\title{
NUMERICAL STUDY OF UNSTEADY FLOW AROUND A CAVITATING HYDROFOIL
}

\author{
M. M. Karim ${ }^{1}$, N. Mostafa ${ }^{2}$ and M. M. A. Sarker ${ }^{3}$
}

${ }^{1}$ Professor, Dept. of Naval Architecture and Marine Engineering, BUET, Dhaka-1000, Email: mmkarim@name.buet.ac.bd

${ }^{2}$ Doctoral Student, Environment and Information Science, Yokohama National University, Japan,

Email:mostafa_nur@yahoo.com

${ }^{3}$ Professor, Dept. of Mathematics, BUET, Dhaka-1000, Email: masarker@math.buet.ac.bd

\begin{abstract}
:
This paper presents a numerical study of the non-cavitating and cavitating flow around CAV 2003 hydrofoil. The phenomenon of cavitation is modeled through a mixture model. For the numerical solution of cavitating flow a bubble dynamics cavitation model is used to describe the generation and evaporation of vapor phase. The non-cavitating study focuses on the influence of the turbulence model and different mesh sizes used in the computation. Three turbulence models such as Spalart-Allmaras, Shear Stress Turbulence (SST) $k-\omega$ model, RNG $k-\varepsilon$ with enhanced wall treatment are used to capture turbulent boundary layer along the hydrofoil surface. The results predicted by these models are compared with each other. The cavitating study first presented an unsteady behavior of the partial cavity attached to the foil. Then, an analysis of a supercavitating condition is performed. The predicted results show good agreement with results published by other researchers.
\end{abstract}

Keywords: Cavitation, CAV2003 hydrofoil, turbulence, mixture model,

\begin{tabular}{llll}
\multicolumn{2}{c}{ NOMENCLATURE } & \multicolumn{2}{l}{ Greek symbols } \\
$C_{D}$ & drag coefficient & $\alpha$ & volume fraction \\
$C_{f}$ & Frictional coefficient & $\nabla$ & vector differential operator \\
$C_{p}$ & pressure coefficient & $\mu_{t}$ & turbulent viscosity \\
$C_{1 \varepsilon}$ & turbulence constant & $\bar{v}$ & turbulent kinematic viscosity \\
$C_{2 \varepsilon}$ & turbulence constant & $\gamma$ & surface tension \\
$C e$ & empirical constant & $\rho$ & density \\
$C_{e}$ & empirical constant & $\rho_{l}$ & liquid density \\
$C_{l}$ & drag coefficient & $\rho_{m}$ & mixture density \\
$f_{g}$ & Gas mass fraction & $\sigma$ & cavitation number \\
$f_{v}$ & vapor mass fraction & $\omega$ & specific dissipation \\
$p$ & pressure & $\varepsilon$ & turbulent dissipation rate \\
$P_{v}$ & vapor pressure & $\tau_{i j}$ & shear stress \\
$p_{\infty}$ & system pressure & $\tau_{w}$ & shear stress at the wall \\
$R_{s}$ & vapor generation source term & $\infty$ & free stream \\
$R_{C}$ & vapor condensation source term & &
\end{tabular}

\section{Introduction}

Cavitation in hydraulic machines causes different problems like vibration, increase of hydrodynamic drag, pressure pulsation, and change in flow kinematics, noise and erosion of solid surface. Most of these problems related to transient behavior of cavitation structure. Cavitation erosion is strongly related to unsteady fluctuations of the cavitation zone. Hence a study of unsteady cavitation behavior is essential for a good 
prediction of the problem. To investigate cavitation phenomena and validate numerical procedures, a number of investigations were performed in the past by Kubota et al. (1992), Alajbegovic et all. (1999), Stutz et al. (2000), Schnerr et al. (2001), and Frobenius et al. (2003). In the last decade various methods for numerical simulation of cavitating flow were developed. Most of the studies treat the two phase flow as a single vapor-liquid phase mixture flow. The evaporation and condensation can be modeled with different source terms that are usually derived from the Rayleigh-Plesset bubble dynamics equation. This approach was first made by Kubota et al. who used the linear part in the Rayleigh-Plesset equation to describe the evaluation of bubble radius as a function of the surrounding pressure. Other cavitation models which included more complex relation between pressure and bubble radius were derived from the Rayleigh-Plesset equation by Schnerr et al. (2001) and Frobenius et al. (2003), but they all include some quantities (like bubble number density and initial bubble diameter ) which are very hard to determine. For example the recommended value for bubble number density that has to be included in the mentioned model is $10^{4} \mathrm{~m}^{-3}$ according to Kubota et al., $10^{8} \mathrm{~m}^{-3}$ according to Schnerr and Sauer or to Frobinus et al and even $10^{12} \mathrm{~m}^{-3}$ according to Alasbegovic et al.

Recently, Singhal et al. (2002), Kunz et al. (1999) and Merkel et al. (1998) proposed to consider a transport equation model for the void ratio, with vaporization/condensation source terms to control the mass transfer between the two phases. This method has the advantage that it can take into account the time influence on the mass transfer phenomena through empirical laws for the source term. It also avoids using quantities like bubble number density and initial bubble diameter. The other way to model cavitation process is by the so called barotropic state law that links the density of vapor-liquid mixture to the local static pressure. The model was proposed by Delannoy and Kueny (1990) and later widely used by S Song et al. (1998), Hofmann et al. (1999), Lohrberg et al. (2002) and Coutier-Delgosha et al. (2003). The results obtained with the barotropic cavitation model show very good correlation to the experiments but the past simulations lacked in robustness of the numerical algorithms, which resulted in numerical instability and sometimes, poor convergence.

A cavitation model, based on bubble dynamics equation proposed by Singhal et al. (2002) is used for computation of cavitating flows. The non-cavitating operation is first characterized in details as a reference for cavitating conditions. Influences of the mesh and turbulence model are studied, mainly by comparing the values of lift and drag. Then, two cavitating conditions are separately analyzed: $\sigma=0.8$ where an unsteady partial cavitating behavior is obtained and $\sigma=0.4$ where a supercavitating flow is observed.

\section{Numerical Simulation}

The numerical model uses an implicit finite volume method associated with multiphase and cavitation model. For numerical simulation of cavitating flow, a bubble dynamics cavitation model is used to describe the cavity formation. The RNG k- $\varepsilon$ turbulence model with enhanced wall treatment is used as a turbulence model. The Reynolds number $\left(R e=5.9^{\times} 10^{5}\right)$ based on chord length is used .The corresponding $y+$ is 5-15. A second order central scheme is used for discretization for space except for the convective terms. The convective term in the momentum equation is discretized by the QUICK scheme for non cavitating flow and second order implicit scheme is used for cavitating problem. Pressure based solver SIMPLE is used as the velocity pressure-coupling algorithm

\section{Multiphase Model}

A single fluid (mixture model) approach is used. The basic approach consists of using standard (Navier-Stokes) viscous flow equation and conventional turbulent model (RNG $k-\varepsilon$ model). The mass and momentum conservation equations (Eq.3 \& 4) together with the transport equation (Eq.5) and the equation of the turbulence model from the set of equations from which fluid density (which is the function of the vapor mass fraction $f_{v}$ ) is computed

The $\rho_{m}-f_{v}$ (mixture density-vapor mass fraction) relation is obtained by Dular et al. (2005):

$$
\frac{1}{\rho}=\frac{f_{v}}{\rho_{v}}-\frac{1-f_{v}}{\rho_{l}}=0
$$


The volume fraction of the vapor phase $\left(\alpha_{v}\right)$ is related to the mass fraction of the vapor phase with:

$\alpha_{v}=f_{v} \frac{\rho_{m}}{\rho_{v}}$

The mass conservation equation for the mixture is:

$$
\frac{\partial}{\partial t}\left(\rho_{m}\right)+\nabla \cdot\left(\rho_{m} \vec{v}_{m}\right)=0
$$

The momentum conservation equation for the mixture is

$$
\frac{\partial}{\partial t}\left(\rho_{m} \vec{v}_{m}\right)+\nabla \cdot\left(\rho_{m} \vec{v}_{m} \vec{v}_{m}\right)=-\nabla p+\nabla \cdot\left[\mu_{m}\left(\nabla \vec{v}_{m}+\nabla \vec{v}_{m}^{T}\right)\right]+\rho m \vec{g}+\vec{F}
$$

And the transport equation for the vapor is:

$$
\frac{\partial}{\partial t}\left(\rho_{m} f_{v}\right)+\nabla \cdot\left(\rho_{m} \vec{v}_{m} f_{v}\right)=R_{e}-R_{c}
$$

\section{Cavitation Model}

The working fluid is assumed to be a mixture of liquid, liquid vapor and noncondensible gas. The vapor mass fraction is the dependent variable in the transport equation. Source terms $R_{e}$ and $R_{c}$ that are included in the transport equation define vapor generation (liquid evaporation) and vapor condensation, respectively. Source terms are the function of the local flow condition (Static pressure, velocity) and fluid properties (liquid and vapor phase densities, saturation pressure and liquid vapor surface tension). The source terms are derived from the Rayleigh-Plesset equation where higher order terms and viscosity term have been left out. The source terms can be expressed as-

$$
\begin{array}{ll}
R_{e}=C_{e} \frac{\sqrt{k}}{\gamma} \rho_{l} \rho_{v} \sqrt{\frac{2}{3} \frac{p_{v}-p}{\rho_{l}}\left(1-f_{v}-f_{g}\right)}, & \text { when } p<p_{v} \\
R_{c}=C_{e} \frac{\sqrt{k}}{\gamma} \rho_{l} \rho_{l} \sqrt{\frac{2}{3} \frac{p_{v}-p}{\rho_{l}} f_{v}}, & \text { when } p>p_{v}
\end{array}
$$

where $C_{e}$ and $C_{c}$ are empirical constants, and $\mathrm{k}$ is the local kinetic energy, $\gamma$ surface tension, $f_{v}$ vapor mass fraction and $f_{g}$ mass fraction of noncondensable(dissolve) gases. $C_{e}$ and $C_{c}$ were determined by comparing experimental and numerical results at different combination of initial conditions and geometries and their values are 0.02 and 0.01 respectively.

\section{Geometry and Computational Domain}

The section of the hydrofoil is presented in Fig. 1 which shows a schematic view of the CAV2003 hydrofoil geometry. The hydrofoil is placed at an $7^{0}$ angle of attack. The equation of the upper surface of the symmetric foil geometry is provided

$$
\frac{y}{c}=a_{0} \sqrt{\frac{x}{c}}+a_{1}\left(\frac{x}{c}\right)+a_{2}\left(\frac{x}{c}\right)^{2}+a_{3}\left(\frac{x}{c}\right)^{3}+a_{4}\left(\frac{x}{c}\right)^{4}
$$

where $a_{0}=0.11858, a_{1}=-0.02972, a_{2}=0.00593, a_{3}=-0.07272, a_{4}=-0.002207$

$\bar{y}=y / c$ and $\bar{x}=x / c$ is the dimensionless coordinate along the chord line. The flow field around the hydrofoil is modeled in two dimensions. The flow from left to right with the hydrofoil of chord length $c=0.1 \mathrm{~m}$ 
submersed in an incompressible fluid is considered. The computational domain is sketched in Fig. 1. The hydrofoil is located at the middle of a channel of length 10c and height 4c.

Fig. 1 shows the total 2D computational domain and boundary conditions. The inlet boundary condition is specified velocity inlet with a constant velocity profile. Upper and lower boundaries are slip walls, i.e., symmetry boundary condition. The outlet uses a constant pressure boundary condition. The foil itself is a no-slip wall, i.e., $\mathrm{u}=0, \mathrm{v}=0$ at the foil surface. In the physical domain the flow is not confined. Nevertheless, a fictitious external rectangular boundary is needed at a large distance from the hydrofoil in order to solve the governing equations numerically. Also the flow at exit is treated as a pressure outlet. The problem setup together with the important dimensions is shown in Fig. 1.

Symmetry

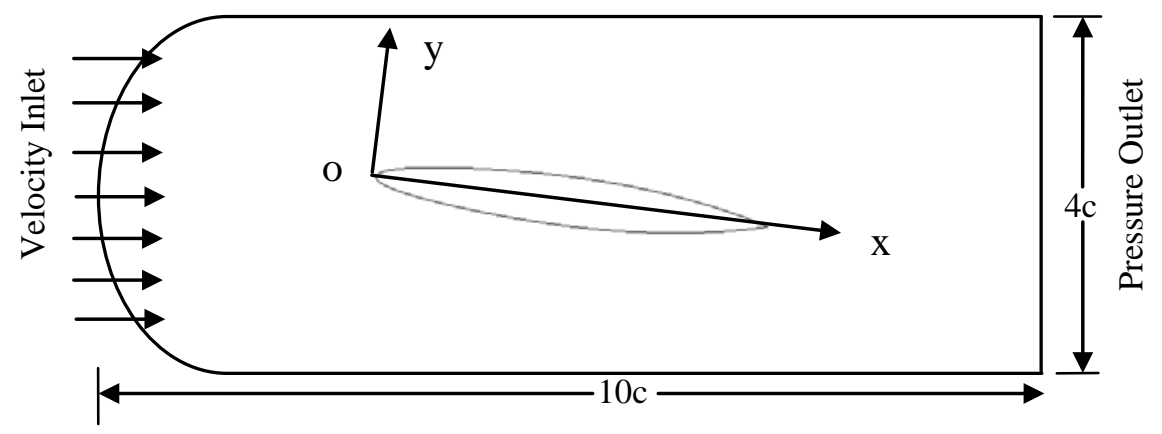

Fig. 1: Schematic Diagram of the Flow Field around CAV2003 Hydrofoil with Boundary condition

\section{Non-cavitating Analysis}

This section will present the methodology that has been applied to select the mesh and turbulence model used for the cavitating computations. The comparisons will be based on convergence criteria and predicted values of lift and drag.

(a)

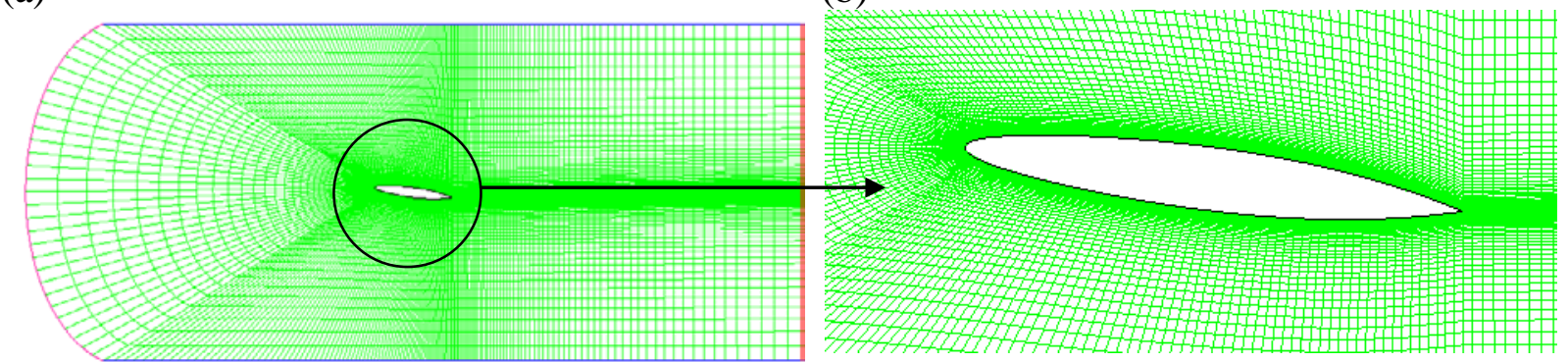

Fig. 2: Grid Lines in Mesh: a) Overall View b) Close-up View Near the Hydrofoil.

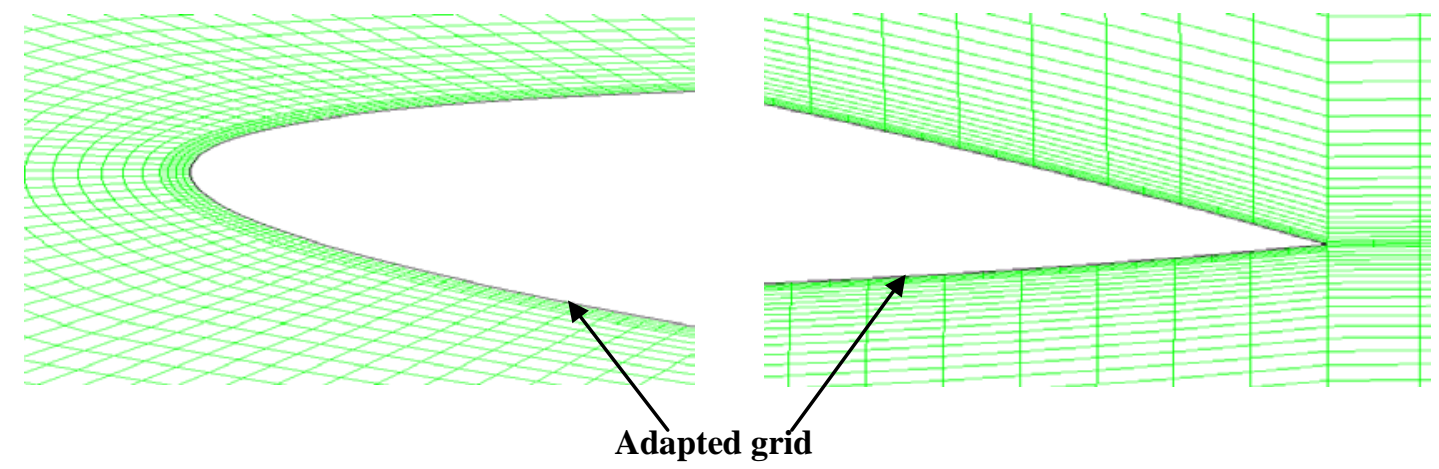

Fig.3: Close up view of the Grids around the Leading edge and Trailing edge of the CAV2003 Hydrofoil 


\subsection{Influence of Mesh}

The C-grid topology is adopted, and the boundary condition used in the simulations is shown also in Fig. 1 . The total of 7 grids are used to investigate the influence of grid parameters such as the number of control volumes, the minimum grid spacing around the hydrofoil on lift force and drag force. A typical grid (GRID 7) is shown in Fig. 2. Most of the cells are located around the foil and a contraction of the grid is applied in its upstream part to obtain an especially fine discretization of the areas where cavitation is expected.

First of all to minimize the CPU time, we tried to create a mesh topology that would allow a coarse grid in the region near the hydrofoil. The first configuration is rejected due to lack of required minimum spacing near the hydrofoil. So we increase the number of grids around the hydrofoil specially at the leading edge to achieve accuracy comparable to the result of Pouffary et al. (2003), Coutier-Delgosha et al. (2003), Yoshinori et al. (2003) and Kawamura et al. (2003). Using all of six configurations, required $y^{+}$and accuracy of result are not achieved. Since Grid 1-6 do not provide good results, adaptation of region near the hydrofoil surface is performed since more cells are needed near the hydrofoil surface to obtain results with high accuracy. The values of the parameters and the computed drag and lift coefficients are summarized in Table 1.

Table 1: Parameter for the grid study

\begin{tabular}{|c|c|c|c|c|c|c|c|}
\hline $\begin{array}{c}\text { Grid } \\
\text { no. }\end{array}$ & $\begin{array}{c}\text { No of } \\
\text { Cells }\end{array}$ & $\begin{array}{c}\text { No of } \\
\text { faces }\end{array}$ & $\begin{array}{c}\text { No of } \\
\text { nodes }\end{array}$ & $\begin{array}{c}\text { Cell } \\
\text { distance }\end{array}$ & $\mathrm{Y}^{+}$ & $\begin{array}{c}\text { Lift force } \\
\text { coefficient }\end{array}$ & $\begin{array}{c}\text { Drag force } \\
\text { coefficient }\end{array}$ \\
\hline 1 & 9912 & 20050 & 10138 & $4.0 \mathrm{e}-04$ & $40-110$ & 0.576 & 0.0375 \\
\hline 2 & 12032 & 24310 & 12278 & $2.5 \mathrm{e}-04$ & $40-90$ & 0.605 & 0.0329 \\
\hline 3 & 13500 & 27260 & 13760 & $2.0 \mathrm{e}-04$ & $25-80$ & 0.611 & 0.0301 \\
\hline 4 & 13974 & 28213 & 14239 & $1.75 \mathrm{e}-04$ & $20-70$ & 0.617 & 0.0292 \\
\hline 5 & 15984 & 32257 & 16273 & $1.25 \mathrm{e}-04$ & $20-60$ & 0.645 & 0.0266 \\
\hline 6 & 16610 & 33515 & 16905 & $1.2 \mathrm{e}-04$ & $15-40$ & 0.658 & 0.0235 \\
\hline 7 & 17684 & 35844 & 18160 & $6.0 \mathrm{e}-05$ & $5-15$ & 0.665 & 0.0244 \\
\hline
\end{tabular}

All the variables are non-dimensionalized with respect to the chord length, the free stream velocity and the density of water unless otherwise mentioned. The computed lift and drag coefficients are found to be very sensitive to the minimum grid spacing at the leading edge. The adapted grid near the foil leading edge and the trailing edge are shown in Fig. 3. Fig. 4 shows the grid sensitivity to the surface pressure distribution on the foil. Three typical mesh densities are chosen for analysis of grid sensitivity. We consider Grid-1, Grid-4 and Grid-7 as coarse, medium and fine mesh respectively. Small difference exists in pressure distribution with medium and fine mesh but there is a large discrepancy for using coarse mesh. The peak of the negative pressure for the fine mesh near the leading edge is higher than those of other two. It is noted that the value of $C p$ at the stagnation point on the nose of the foil become much closure to 1. Fig. 5 shows the comparison of pressure distribution on the foil surface that reveals good agreement with the result of Kawamura et al. (2003).

Using Grid-7 with 17684 cells and comparing with the results of Kawamura, it is observed that this grid system provides sufficiently good results. Here the minimum grid spacing from the wall is $6 \times 10^{-5}$ that is lower than the recommended value, $1 \times 10-4$. The corresponding $y+$ is $5-15$. Based on these results, the adapted grid, i.e., Grid-7 is finally chosen for the simulations of cavitating flow.

\subsection{Influence of Turbulence Model}

To simulate non cavitating flow there exists a great influence of different turbulence models. Here, we simulate the non-cavitating flow with different turbulence models such as RNG k- $\varepsilon$ with enhanced wall Treatment, Spalart- Allmaras and the $k-\omega$ Shear Stress Turbulence model. The comparison among the different models is made on the basis of the predicted values of lift and drag coefficients as shown in Table 2a \& 2b in which the viscous and pressure parts are analyzed separately. The results show a good agreement with Spalart- Allmaras and $k-\varepsilon$ with enhanced wall treatment. The $k-\omega$ model predicts a value that is lower than other model. The differences are due to the calculation of a separated flow near the trailing edge. 
In Table 3 computed lift and drag coefficient for the non cavitating flow with turbulence model k- $\varepsilon$ is compared with the results of Pouffary et al. (2003) and Courtier-Delgosha et al. (2003) and $k$ - $\omega$ model with Kawamura et al. (2003). The present result shows a good agreement on the prediction of the total lift especially with CourtierDelgosha et al (2003). Finally, we decided to use the $k-\varepsilon$ model with enhanced wall treatment for cavitating calculations.

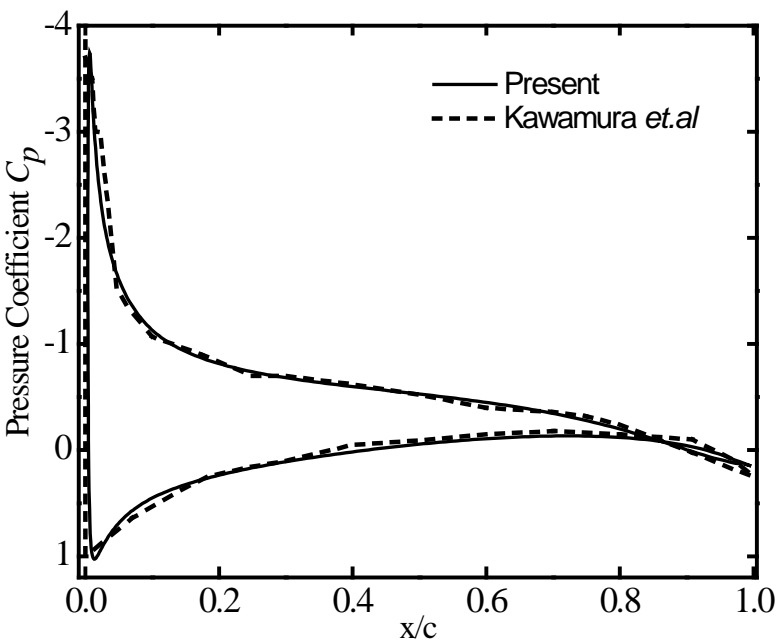

Fig.4:Grid sensitivity to surface pressure distribution for non-cavitating flow over CAV2003 Hydrofoil

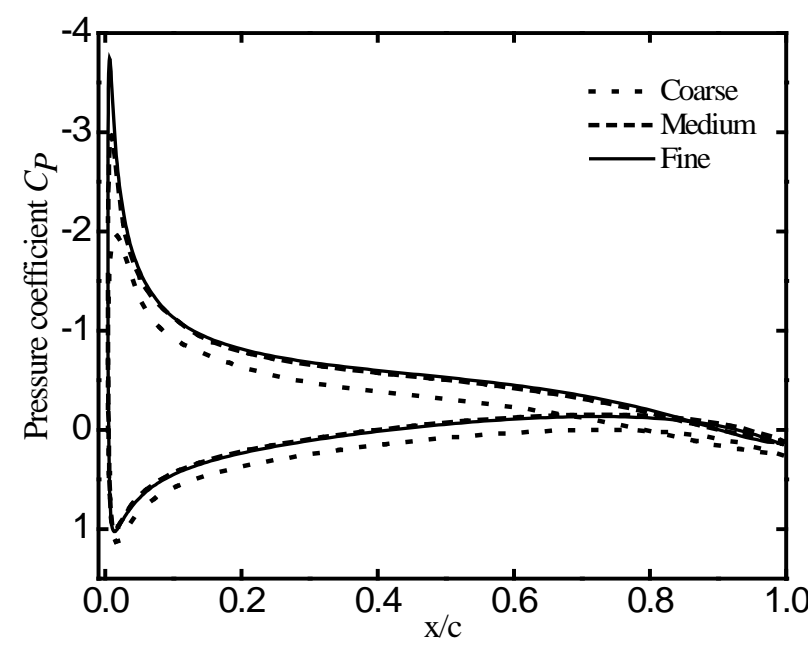

Fig.5:Comparison of the pressure coefficient on the foil surface in non-cavitating condition

Table 2a: Turbulence effect on lift

\begin{tabular}{|l|l|l|l|}
\hline \multirow{2}{*}{ Models } & \multicolumn{3}{|c|}{ Lift coefficient } \\
\cline { 2 - 4 } & Viscous & Pressure & Total \\
\hline$k-\omega$ & -0.0005 & 0.5994 & 0.5989 \\
\hline Spalart-Allmaras & -0.0004 & 0.6522 & 0.6518 \\
\hline$k-\varepsilon$ with enhanced wall treatment & -0.0004 & 0.6567 & 0.6564 \\
\hline
\end{tabular}

Table 2b: Turbulence effect on drag

\begin{tabular}{|l|l|l|l|}
\hline \multirow{2}{*}{ Models } & \multicolumn{3}{|c|}{ Drag coefficient } \\
\cline { 2 - 4 } & Viscous & Pressure & Total \\
\hline$k-\omega$ & 0.01061 & 0.01061 & 0.0212 \\
\hline Spalart-Allmaras & 0.00886 & 0.02223 & 0.0311 \\
\hline$k-\varepsilon$ with enhanced wall treatment & 0.01013 & 0.01433 & 0.0245 \\
\hline
\end{tabular}

\section{Cavitating Condition}

This section presents results computed for the typical cavitation numbers $\sigma=0.8$ and $\sigma=0.4$. For simulation the convergence criterion is determined by observing the evaluation of different flow parameters (velocity magnitude at inlet, static pressure behind the hydrofoil) in the computational domain. For computation, each value of residual is taken as $10^{-4}$. Time step size has a great influence on simulation of cavitating flow. Different time step values are tested, eventually the time step for unsteady computation is set to $5 \times 10^{-5}$ and approximately 30 iterations per time step are needed to obtain a converged solution. The value $\rho_{l}=998.2 \mathrm{~kg} / \mathrm{m} 3$, $\rho_{v}=0.5542 \mathrm{~kg} / \mathrm{m} 3, \mu_{l}=10^{-3}$ pas, $\mu_{1}=1.34 \times 10^{-5}$ pas, $\quad \gamma=0.0717 \mathrm{~N} / \mathrm{M}$ for liquid and vapor density, liquid and vapor dynamical viscosity and surface tension respectively are used for simulation. To predict the behavior of the cavitating flow for the values of cavitation number $\sigma=0.8$ and $\sigma=0.4$, we first present comparisons of the computed time-averaged lift and drag coefficient for cavitating flow with Pouffary et 
al. (2003), Courtier -Delgosha et al. (2003), Kawamura et al. (2003) and Yoshinori et al. (2003). Table 4 shows that the lift coefficient and the drag coefficient are in good agreement with published results.

Table 3: Comparison of $\bar{C}_{L}$ and $\bar{C}_{D}$ for non cavitating case

\begin{tabular}{|l|l|l|}
\hline & $\bar{C}_{L}$ & $\bar{C}_{D}$ \\
\hline Present & 0.656 & 0.0245 \\
\hline Pouffary & 0.622 & 0.0294 \\
\hline Courtier-Delgosha & 0.66 & 0.015 \\
\hline Kawamura & 0.64 & 0.018 \\
\hline
\end{tabular}

Table 4: Comparisons of time-averaged lift and drag coefficient at cavitation numbers $\sigma=0.8$ and $\sigma=0.4$

\begin{tabular}{|l|l|l|l|l|}
\hline \multirow{2}{*}{} & \multicolumn{2}{|c|}{$\sigma=0.8$} & \multicolumn{2}{c|}{$\sigma=0.4$} \\
\cline { 2 - 5 } & $\bar{C}_{L}$ & $\bar{C}_{D}$ & $\bar{C}_{L}$ & $\bar{C}_{D}$ \\
\hline Present & 0.44 & 0.077 & 0.214 & 0.076 \\
\hline Pouffary & 0.4566 & 0.0783 & 0.291 & 0.086 \\
\hline Courtier-Delgosha & 0.450 & 0.0700 & 0.200 & 0.065 \\
\hline Kawamura & 0.399 & 0.047 & 0.187 & 0.063 \\
\hline Yoshinori & 0.417 & 0.0638 & 0.160 & 0.0568 \\
\hline
\end{tabular}

The time average value of lift and drag coefficients calculated by present method for the cavitation number $\sigma=$ 0.8 are very close to the numerical result of Pouffary et al. (2003). However, the results show little discrepancy at cavitation number $\sigma=0.4$. This discrepancy may be attributed due to the fact that different researchers used different turbulence models. The comparisons of the pressure distribution on the foil surface for $\sigma=0.4$ is shown in Fig. 6. It compares the present result with the result of Kawamura et al. (2003). There exists a good agreement but some difference in magnitude may be due to the $k$ - $\omega$ turbulence model used by Kawamura et al. (2003).The difference in pressure distribution on the face side is found very small. Similar comparison is shown in Fig. 6 for $\sigma=0.8$. The time history of the lift and drag coefficient computed by mixture model at $\sigma=0.8$ and $\sigma$ $=0.4$ are shown in Fig. $7 \& 8$ respectively. The characteristics of the curve of lift and drag coefficients are almost similar. The contours of pressure coefficient for cavitation numbers $\sigma=0.8$ and 0.4 are shown in Fig. 9 .
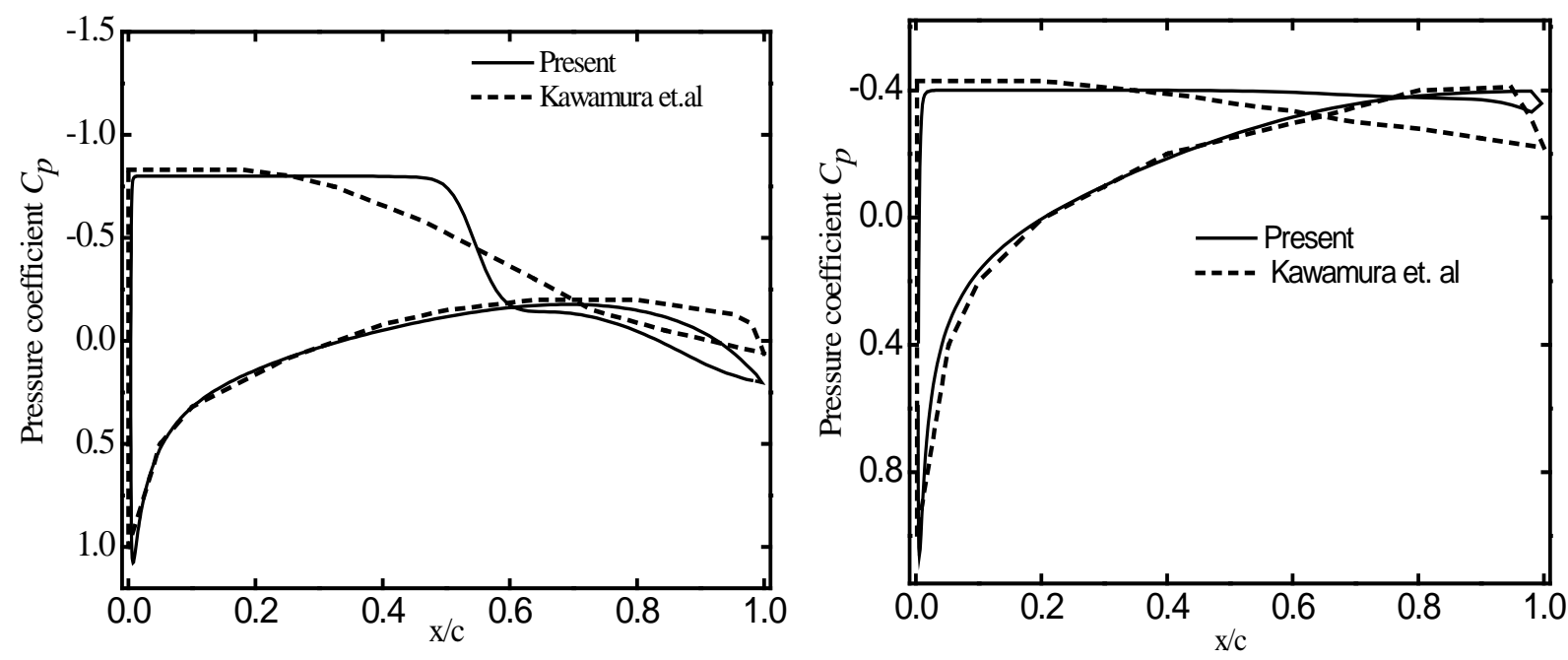

Fig. 6: Comparison of the pressure coefficient on the foil surface at $\sigma=0.8$ and 0.4 respectively 

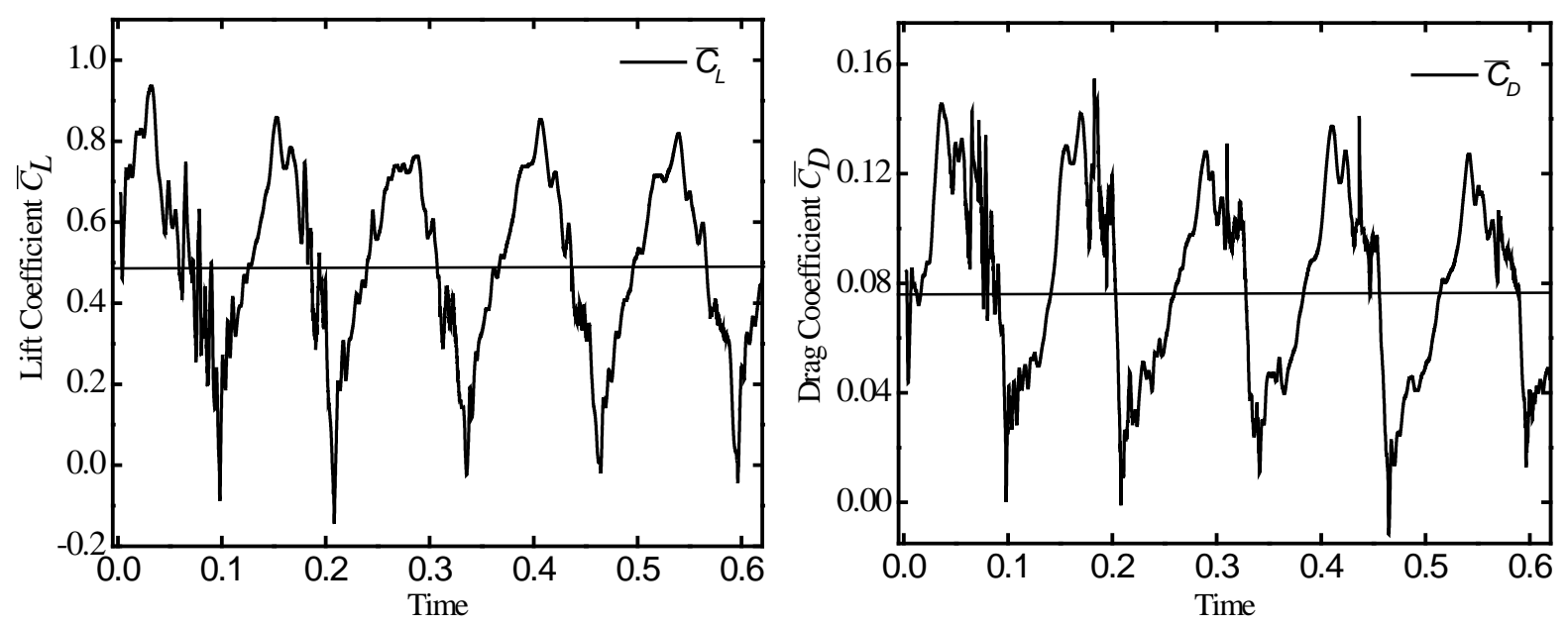

Fig. 7: Time history of Lift coefficient and drag coefficient at $\sigma=0.8$
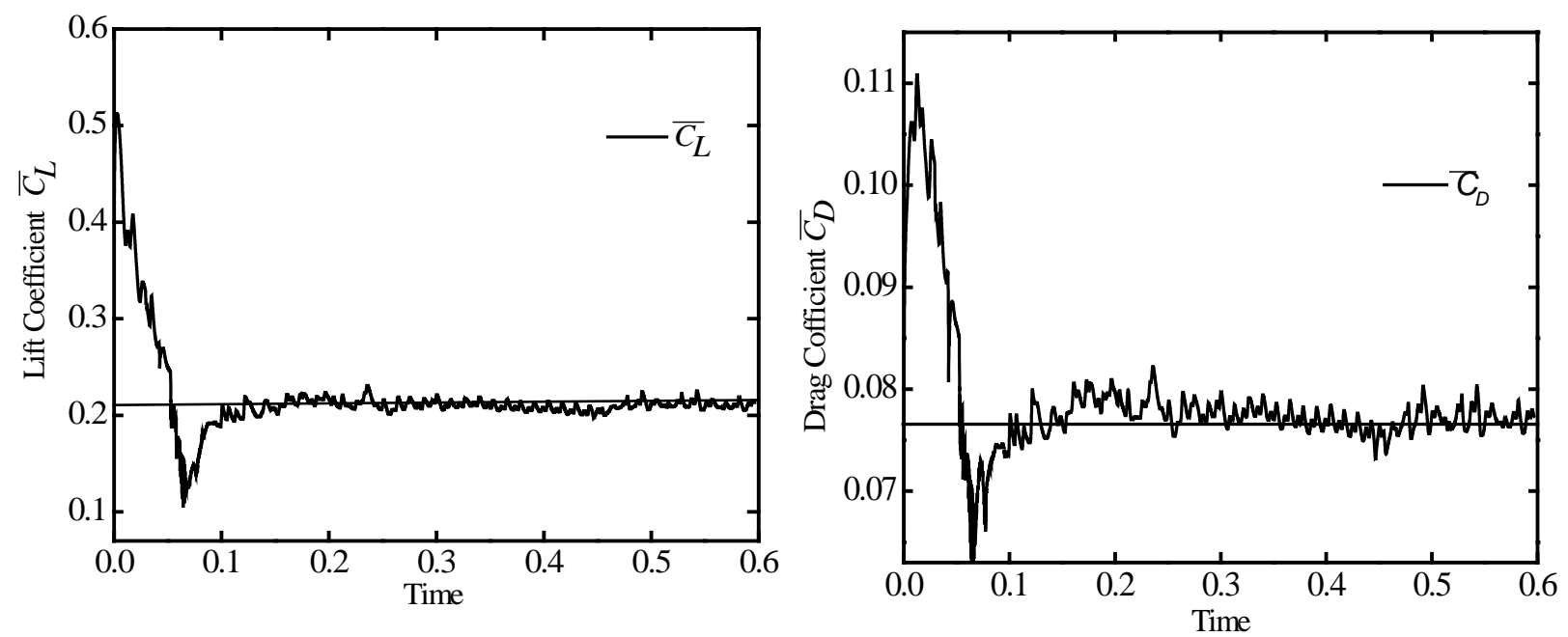

Fig. 8: Time history of a) lift coefficient and b) drag coefficient at $\sigma=0.4$

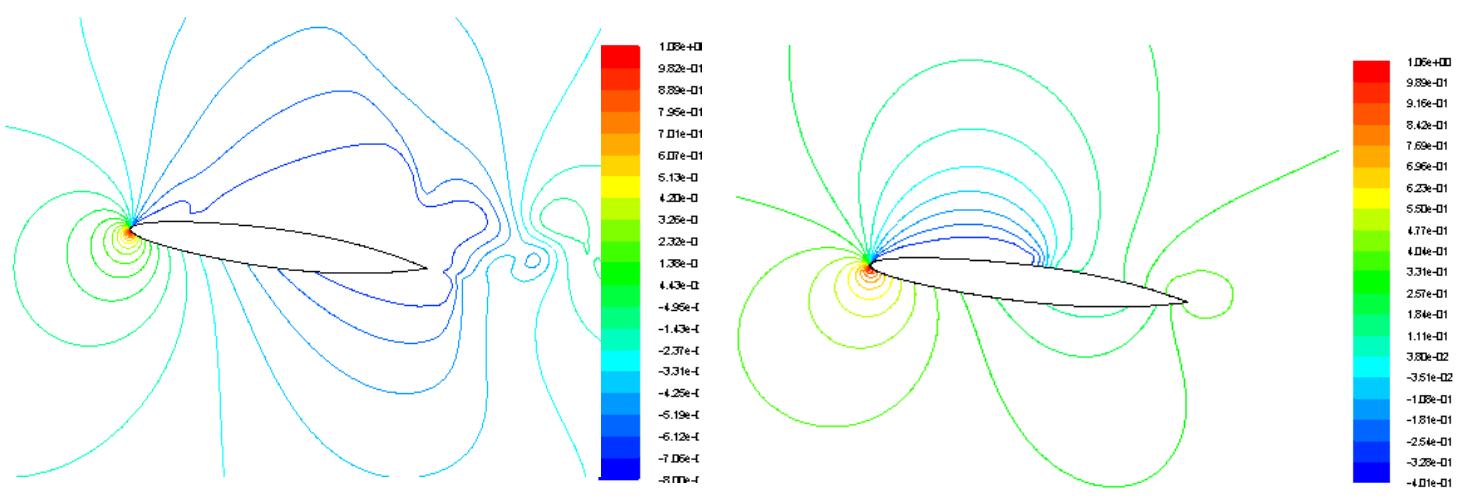

Fig. 9: Contours of pressure coefficient at $\sigma=0.8$ and 0.4 respectively

These contours show the expansion of cavity and their sizes for different cavitation number. Fig. 10 shows vapor volume fraction for cavitation number $\sigma=0.8 \& 0.4$ respectively. At $\sigma=0.8$ the half of the hydrofoil is 
covered with vapor. At $\sigma=0.4$ the back surface is covered with vapor and it decreases with the increase in cavitation number and cavity length become shorter
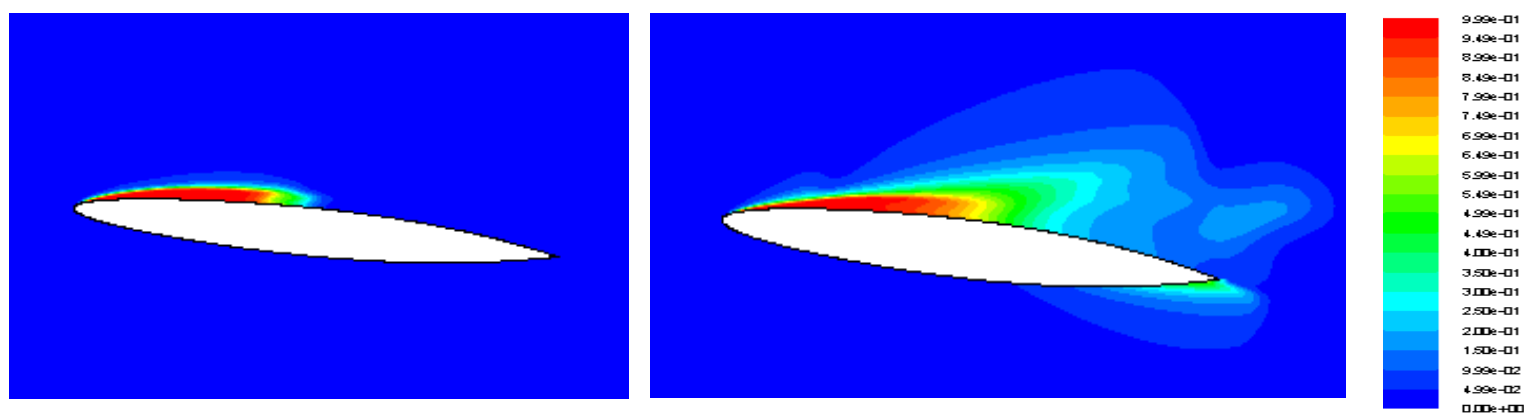

Fig. 10: Contours of vapor volume fraction at $\sigma=0.8$, and 0.4 respectivly

\section{Conclusion}

Two-dimensional finite volume method has been applied to simulate incompressible flow around CAV2003 hydrofoil. Three turbulence models such as Spalart-Allmaras, RNG $k-\varepsilon$ with enhanced wall treatment and SST $k-\omega$ models are used to capture boundary layer in the simulation of steady flow around hydrofoil. It is observed that the RNG $k-\varepsilon$ model with enhanced wall treatment and Spalart-Allmaras model compute the lift coefficient accurately. However, only RNG $k-\varepsilon$ model with enhanced wall treatment is used for simulation of cavitating flow because of its better performance.

The results obtained by simulating an unsteady partial cavitating behavior at $\sigma=0.8$ are very promising. The flow is very well predicted by $k-\varepsilon$ turbulence model. Especially the cavity length is calculated with a high degree of accuracy. The simulation at cavitation number $\sigma=0.4$ predicted a stable supercavitation behavior. Super cavities starting from the vicinity of the foil leading edge and exceed the chord length of the hydrofoil. For simulation of cavitating flow, a time step size $5 \times 10^{-5} \mathrm{~s}$ seems to allow the development of unsteadiness, which highlights the crucial importance of that parameter for the triggering of cavitation instabilities. Computed values of lift and drag coefficient of both the cases agree satisfactorily with published results.

\section{References}

Alajbegovic, A., Groger, H. A. and Philipp, H., (1999): Calculation of transient cavitation in nozzle using the two-fluid model, in: 12th Annual Conference on Liquid Atomization and Spray Systems, Indianapolis, IN, USA. Coutier-Delgosha, O., Fortes-Patella, R. and Reboud, J.L., (2003): Evaluation of turbulence model influence on the numerical simulations on unsteady cavitation, J. Fluids Engrg. 125, 38-45.doi:10.1115/1.1524584

Coutier-Delgosha, O. and Jacques Andre Astolfi, (2003): Numerical reduction of cavitating flow on a twodimensional symmetrical hydrofoil with a single fluid model, Fifth International Symposium on Cavitation(Cav2003), Osaka, Japan, November 1-4.

Delannoy, Y. and Kueny, J. L., (1990): Two phase flow approach in unsteady cavitation modelling, in: Cavitation and Multiphase Flow Forum, ASMEFED, vol. 98, pp. 153-158

Dular, M., Bacher, R., Stoffel, B. and Širok, B. (2005): Experimantal evaluation of numerical simulation of cavitating flow around hydrofoil, Europian Journal of Machanics B/Fluids 24, 522-538.

Frobenius, M., Schilling, R., Bachert, R. and Stoffel, B., (2003): Three-dimensional, unsteady cavitation effects on a single hydrofoil and in a radial pump - measurements and numerical simulations, Part two: Numerical simulation, Proceedings of the Fifth International Symposium on Cavitation, Osaka, Japan.

Hofmann, M., Lohrberg, H., Ludwig, G., Stoffel, B., Reboud, J.L. and Fortes-Patella, R., (1999): Numerical and experimental investigations on the self - oscillating behaviour of cloud cavitation - Part 1: Visualisation, Proceedings of the 3rd ASME/JSME Joint Fluids Engineering Conference, San Francisco, CA.

Kubota, A., Hiroharu, K. And Yamaguchi, H., (1992): A new modelling of cavitating flows, a numerical study of unsteady cavitation on a hydrofoil section, J. Fluid Mech. 240, 59-96. doi:10.1017/S002211209200003X

Kunz, R., Boger, D., Chyczewski, T., Stinebring, D. And Gibeling, H., (1999): Multi-phase CFD analysis of natural and ventilated cavitation about submerged bodies, ASME FEDSM99-7364, San Francisco. 
Kawamura, T., Sakuda, M., (2003): Comparison of bubble and sheet cavitation models for simulation of cavitation flow over a hydrofoil, Fifth International Symposium on Cavitation(Cav2003), Osaka, Japan, November 1-4.

Lohrberg, H., Stoffel, B., Fortes-Patella, R. and Reboud, J. L., (2002): Numerical and experimental investigations on the cavitation flow in cascade of hydrofoils, Exp. Fluids 33 (2002) 578-586. Merkle, C.L., Feng, J. and Buelow, P.E.O (1998): Computational modelling of the dynamics of sheet cavitation, in: 3rd Int. Symp. on Cavitation,Grenoble, France.

Pouffary, B., Fortes-Patela, R., Reboud, J.L., (2003): Numerical simulation of cavitating flow around a 2D hydrofoil: A barotropic approach, Fifth International Symposium on Cavitation (Cav2003), Osaka, Japan, November 1-4.

Stutz, B. and Reboud, J. L., (2000): Measurements within unsteady cavitation, Exp. Fluids 29, 545-552. doi:10.1007/s003480000122

Schnerr, G. H. and Sauer, J., (2001): Physical and numerical modelling of unsteady cavitation dynamics, 4th International Conference on Multiphase Flow, ICMF-2001, New Orleans, USA.

Singhal, A. K., Li, H., Atahavale, M.M. and Jiang, Y., (2002): Mathematical basis and validation of the full cavitation model, J. Fluid Engg. 124 (2002) 617-624.

doi:10.1115/1.1486223

S Song, C. C. and He, J., (1998): Numerical simulation of cavitating flows by single-phase flow approach, 3rd International Symposium on Cavitation, Grenoble, France, pp. 295-300.

Yoshinori S., Ichiro N., Tosshiaki I., (2003): Numerical analysis of unsteady vaporous cavitating flow around a hydrofoil, Fifth International Symposium on Cavitation(Cav2003), Osaka, Japan, November 1-4. 